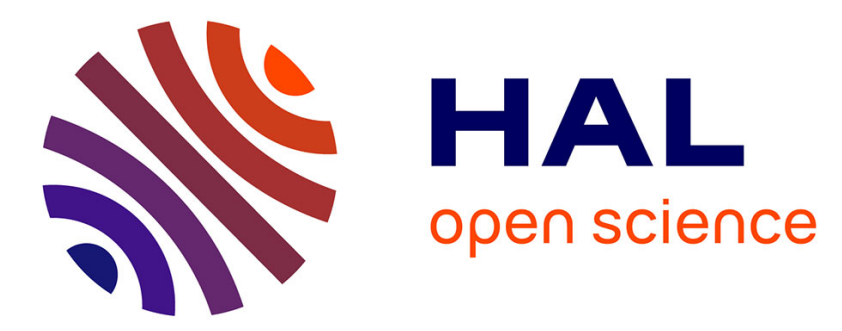

\title{
Experimental analysis of the transverse mechanical behaviour of annulus fibrosus tissue
}

Adrien Baldit, Dominique Ambard, Fabien Cherblanc, Pascale Royer

\section{To cite this version:}

Adrien Baldit, Dominique Ambard, Fabien Cherblanc, Pascale Royer. Experimental analysis of the transverse mechanical behaviour of annulus fibrosus tissue. Biomechanics and Modeling in Mechanobiology, 2014, 13 (3), pp.643-652. 10.1007/s10237-013-0524-x . hal-00862690

\section{HAL Id: hal-00862690 \\ https://hal.science/hal-00862690}

Submitted on 17 Sep 2013

HAL is a multi-disciplinary open access archive for the deposit and dissemination of scientific research documents, whether they are published or not. The documents may come from teaching and research institutions in France or abroad, or from public or private research centers.
L'archive ouverte pluridisciplinaire HAL, est destinée au dépôt et à la diffusion de documents scientifiques de niveau recherche, publiés ou non, émanant des établissements d'enseignement et de recherche français ou étrangers, des laboratoires publics ou privés. 
Noname manuscript No.

(will be inserted by the editor)

\title{
Experimental analysis of the transverse mechanical behaviour of annulus fibrosus tissue.
}

\author{
A. Baldit - D. Ambard - F. Cherblanc . \\ P. Royer
}

Received: date / Accepted: date

\begin{abstract}
Uniaxial tensile and relaxation tests were carried out on annulus fibrosus samples carved out in the circumferential direction. Images were shot perpendicularly to the loading direction. Digital image correlation techniques accurately measured the evolution of full displacement fields in both transverse directions: plane of fibres and plane of lamellae. In the fibre plane, strains were governed by the reorientation of fibres along the loading direction. This implies strong transverse shrinkage with quasi-linear behaviour. Conversely, a wide range of behaviour was observed in the lamella plane: from shrinkage to swelling. Strong non-linear evolutions were generally obtained. The strain

A. Baldit · D. Ambard · F. Cherblanc · P. Royer

LMGC - UMR 5508, CNRS, University of Montpellier 2, CC 048 Place Eugne Bataillon, 34095 Montpellier cedex 5, France

Tel.: (33) 467-144-681

Fax: (33) 467-144-555

E-mail: adrien.baldit@univ-montp2.fr
\end{abstract}


field in the lamella plane generally presented a central strip section with more pronounced swelling. Our physical interpretation relies on the porous nature of annulus tissue and its anisotropic stiffness. Indeed, the liquid overpressure generated inside the sample by the strong shrinkage in the fibre plane discharges in the perpendicular direction since rigidity is lower in the lamella plane. Regarding the strain field measured in the lamella plane, this interpretation agrees with (a) symmetric strain distribution with respect to the longitudinal axis of samples, (b) the reversal in behaviour from shrinkage to swelling, (c) the decrease in strain during relaxation tests associated with outward flows. The variety of transverse behaviours observed experimentally could result from uncertainties regarding the initial reference state of tissue samples. Since the mechanical behaviour is highly non-linear, experimental results underline that a slight uncertainty concerning the pre-stress applied to samples can lead to wide variability in the mechanical properties identified.

Keywords intervertebral disc - annulus fibrosus - mechanical behaviour . poro-mechanics $\cdot$ digital image correlation

\section{Introduction}

While transmitting the physiological loads along the spine, the inter-vertebral disc (IVD) is subjected to large, multi-directional strains [Costi et al 2007, Woods et al 2010]. The role played by the annulus fibrosus (AF) tissue is to hold the nucleus pulposus (NP) as it undergoes overpressure. Its high mechanical stiffness is ensured by its particular micro-structure, i.e., a woven 
oriented structure of collagen fibres that provides adaptability to multi-axial stresses. Furthermore, collagen fibres are embedded in a highly hydrated matrix. Its large liquid phase volume fraction $(60-70 \%)$ [Rannou et al 2000] facilitates the convective and diffusive solute transport of cell nutrients through its structure. Fluid transport is crucial to disc viscoelastic behaviour and cell nutrition processes [Gu et al 1999, Grunhagen et al 2006, Magnier et al 2009, Malandrino et al 2011].

Many experimental studies have underlined the anisotropic and non-linear mechanical behaviour of AF [Baldit et al 2012, Elliott and Setton 2001,Fujita et al 1997, Guerin and Elliott 2007, Iatridis et al 2005, Klisch and Lotz 1999, Smith et al 2008, Wagner and Lotz 2004]. These were mainly conducted in the framework of uni-axial tensile tests, however few authors have experimentally investigated bi-axial behaviour [Bass et al 2004,Bruehlmann et al 2004]. Various types of modelling of AF mechanical behaviour have been proposed in the context of fibre-reinforced theory. Thanks to their high degree of freedom number, these models have been successfully employed to curvefit much experimental stressstrain data in different configurations: tension or compression on axial, radial or circumferential samples [Guerin and Elliott 2007, Klisch and Lotz 1999, Peng et al 2006, Wagner and Lotz 2004]. Nevertheless, these descriptions fail to represent the hysteresis observed under loading cycles [Ambard and Cherblanc 2009, Baldit et al 2012, Iatridis et al 1997, Tower et al 2002] considered to be related to a viscous component. Transverse strains were generally used to estimate apparent Poisson's ratio while their non-linearities were not really investigated. 
Indeed, IVD tissue can be assimilated to porous media where the liquid phase flow plays a major role in macroscopic mechanical behaviour [Huang et al 2001, Yao and Gu 2007]. This is classically described by a poro-elastic formulation and can account for coupling effects between macroscopic mechanical strains and the viscous flow through it [Ferguson et al 2004, Silva et al 2005, Swider et al 2010, Yao and Gu 2007]. In this framework, the porous structure is usually described as elastic behaviour unable to capture material nonlinearities. A rheological model able to represent both non-linear behaviour and viscous hysteresis has been proposed by [Ambard and Cherblanc 2009]. By accurately representing unidirectional stress-strain curves under loading cycles, this model emphasizes the dependence of the AF mechanical response on liquid phase characteristics. In our view, an appropriate mechanistic model of the IVD cannot avoid taking into account the coupling with the liquid phase.

When attempting to characterize the mechanical behaviour of soft biological tissue, various experimental constraints appear. Indeed, physiological conditions are generally required to preserve the physical properties of material. Thus, samples must be immersed in a thermo-regulated bath during experiments which precludes the use of classical strain measurement techniques. An alternative is to rely on image analysis methods have the advantage of being non-invasive. To compute displacement measurement, image analysis methods need a contrasted pattern to follow markers throughout mechanical testing. In the literature, these tend to be glued markers [Bruehlmann et al 2004, Holzapfel et al 2005, O'Connell et al 2012], pins inserted through tissue thick- 
ness [Bass et al 2004, Wagner and Lotz 2004], stained tissue using laser or chemical baths [Huyghe and Jongeneelen 2012, Michalek et al 2010] or alternatively, digital image correlation performed on natural texture [Guerin and Elliott 2006, Guerin and Elliott 2007, O'Connell et al 2009] or black paint speckle-coated samples [Han et al 2012]. Nevertheless, image analysis is based on surface displacement, thus physical makers and glue on soft tissue may disturb surface behaviour and impact strain measures even if considered negligible. Less invasive works have used grey scale thresholding [Elliott and Setton 2001, Tower et al 2002, Michalek et al 2009] or cell nucleus to investigate micro-structural strains [Bruehlmann et al 2004]. In all cases, the number of markers ranges from 2 to 100 [Elliott and Setton 2001].

The present contribution therefore aims at analysing the mechanical behaviour of AF samples subjected to uni-axial tensile tests by investigating transverse strain fields. They are computed using a digital image correlation (DIC) method. To avoid any perturbation, the natural pattern of AF samples is used to determine the transverse strain fields while the sample is immersed in a thermo-regulated bath. The experimental results underline the anisotropic nature of AF tissue emphasizing that its mechanical behaviour clearly differs from classical poro-elastic description. Correlating the strain fields in both transverse directions highlights coupling effects associated with fluid transfer. Moreover, relaxation tests are used to estimate the hydraulic permeability. This means that the AF is essentially a porous material justifying the introduction of fluid flow characteristics when modelling its mechanical behaviour. 


\section{Materials and Methods}

2.1 Sample preparation

Lumbar discs were harvested from cadavers of domestic pigs $(\approx 160 \mathrm{Kg})$ obtained from a local slaughter house. The lumbar spine was excised and stored in a $-12^{\circ} \mathrm{C}$ freezer. IVD were separated from the vertebral bodies by blunt dissection. Then, posterior, lateral and anterior quadrants were separated from each disc using a surgical knife. From each quadrant, one parallelepiped specimen was carved out using surgical tools (scalpel, scissors) (Fig 1). Thus, all specimens were excised in anatomical cut, parallel to the horizontal plane along the circumferential direction $\mathbf{e}_{\theta}$.

The circumferential length of each specimen, $L_{\theta \theta}$, was limited using a surgical knife to approximately $10 \mathrm{~mm}$. Then, to be fixed to the testing device grips, both specimen ends were glued onto aluminium cylinders using cyano-acrylate adhesive (Fig. 1) [Ambard and Cherblanc 2009, Fujita et al 1997, Fujita et al 2000, Michalek et al 2009, Michalek et al 2010, O'Connell et al 2012,Skaggs et al 1994]. Before testing, specimens were stored in physiological conditions, i.e., in a 0.15 mol. $l^{-1} \mathrm{NaCl}$ solution at $\mathrm{T}=37^{\circ} \mathrm{C}$. Finally, 19 parallelepiped porcine samples $\left(L_{z z} \times L_{r r} \times L_{\theta \theta}=3.78 \pm 0.95 \times 3.67 \pm 0.67 \times 9.77 \pm 1.44 \mathrm{~mm}^{3}\right)$ were prepared. 

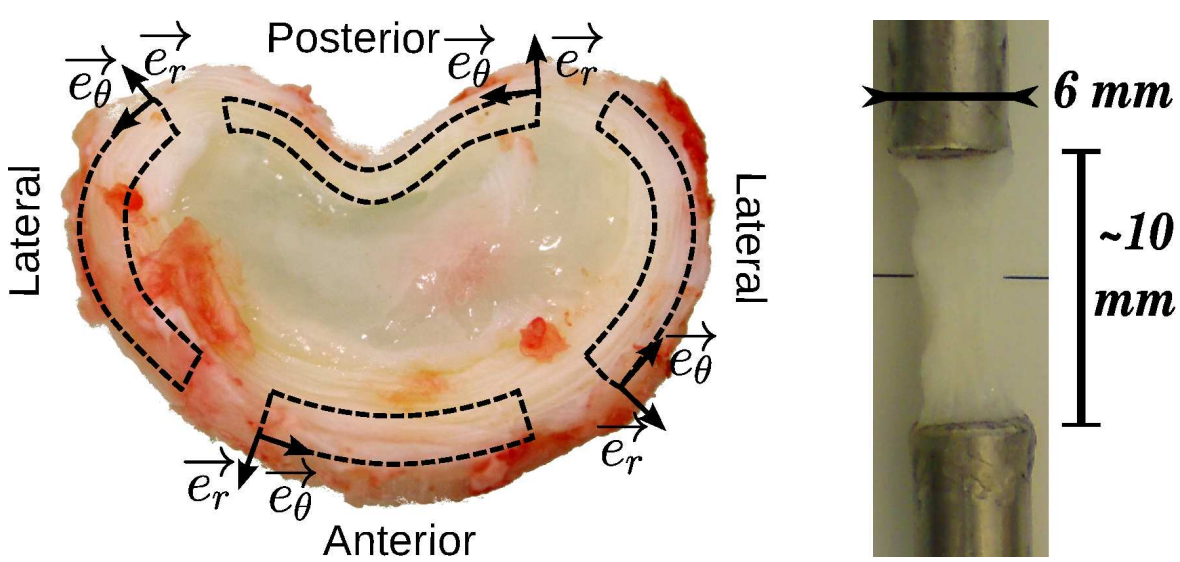

Fig. 1: Specimen extraction and sample

\subsection{Mechanical testing}

Mechanical tests were carried out ex situ in physiological conditions. The testing device was composed of a texture analyser (LF-Plus, Lloyd Instruments) equipped with a $50 \mathrm{~N}$ load cell. Two high resolution cameras (AVT Stingray, 16 bits, $1600 \times 1200$ pixels) were positioned perpendicularly to the tensile direction, $\mathbf{e}_{\theta}$, to observe both transverse planes (Fig. 2). Prime lenses (SchneiderKrevznach xenoplan 2.8/28 - 0901) set on cameras gave a spatial resolution of $3.65 \mu \mathrm{m} / \mathrm{pix}$.

To control the thermo-chemical environment and avoid sample dehydration during experiments, a transparent bath was designed and filled with physiological solution. Two glass strips $\left(L \times W \times T=50 \times 10 \times 1 \mathrm{~mm}^{3}\right)$ were used as windows to decrease optical distortions. The bath was connected to a thermoregulated flow circulation to control the temperature at $T=37^{\circ} \mathrm{C}$ and ensure constant solution level. 
The objective was to perform uniaxial tensile tests. To respect physiological solicitations, we imposed a circumferential strain of $E_{\max }=10 \%$ which has been reported to be the maximum observed strain during physiological motions [Costi et al 2007,Shirazi-Adl 1989]. The experimental procedure carried out with each sample relied on four steps. First of all, the specimen was subjected to a preload of $0.05 \mathrm{~N}$. Second, a conditioning test of 10 loading cycles was performed. During this conditioning test, the maximum imposed strain of $12 \%$ was slightly larger than the targeted strain for tensile testing. This aimed at, on one hand, breaking the fibres damaged during the sample preparation and, on the other hand, controlling the quality of sample ends gluing. Third, the sample was retained left in the bath for one hour so as to reach poromechanical equilibrium. This state was henceforth considered afterwards to be as the reference state at zero strain. The tensile test was then performed. A displacement of $1 \mathrm{~mm}$ was imposed at a velocity of $0.01 \mathrm{~mm} . \mathrm{s}^{-1}$ corresponding to a strain rate of $10^{-3} s^{-1}$. Finally, a relaxation test was carried out by holding the sample at maximum strain for $1000 \mathrm{~s}$. During tensile and relaxation tests, digital images were shot every $4 s$ in both transverse directions, $\mathbf{e}_{z}$ and $\mathbf{e}_{r}$.

2.3 Image analysis method

The image analysis procedure was based on a Digital Image Correlation (DIC) method. Academic software (KelKins) was used and a detailed description of the method can be found in [Wattrisse et al 2001]. The principle is to determine the pixel counterpart of an undistorted image in a corresponding dis- 


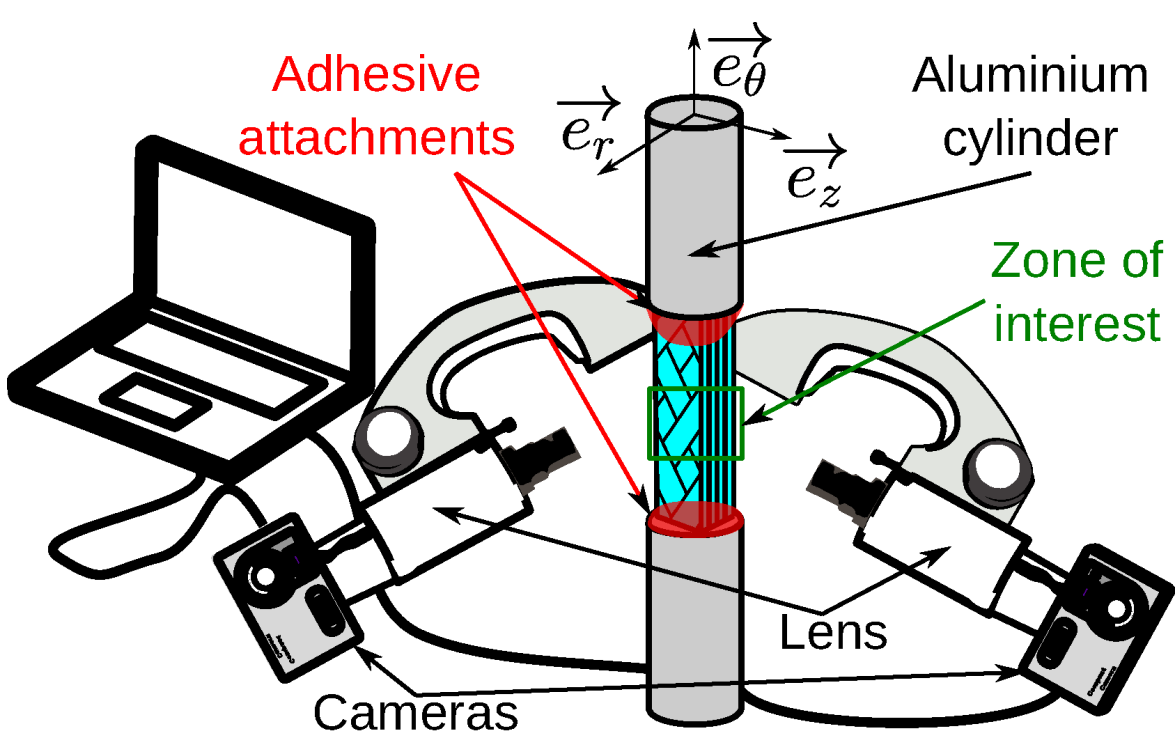

Fig. 2: Tensile test principle

torted one. Therefore, it provides the local displacement field at each point of an initial $0.18 \mathrm{~mm}$ grid. Digital images were focused in the central part of the samples to increase pixel accuracy and avoid side effects produced by the glue bonds on the aluminium grips. This central part represented about $60 \%$ of the total specimen area.

Finally, the strain tensor, E, is computed from the displacement field using a centred finite-difference formulation of the Green-Lagrange definition:

$$
\mathbf{E}=\frac{1}{2}\left(\nabla \mathbf{u}^{T}+\nabla \mathbf{u}+\nabla \mathbf{u}^{T} \cdot \nabla \mathbf{u}\right)
$$

where $\mathbf{u}$ is the local displacement vector and $T$ mean transposed matrix.

When using DIC methods, image contrasts are generally artificially increased using black paint projected onto a white surface [Han et al 2012] or grey scale thresholding [Guerin and Elliott 2006, Guerin and Elliott 2007]. This 
leads to highly contrasted images of randomly disposed pattern. In the case of $\mathrm{AF}$ tissue, a black paint speckled preparation might have disturbed the apparent surface behaviour so we preferred to rely on the weakly contrasted nature of tissue. The use of a 16 bit camera was an alternative to numerically increase the natural contrast. Furthermore, the moving windows were widened to $0.18 \times 0.18 \mathrm{~mm}^{2}$ to provide better, more discriminating correlation indicators.

The optical path crossed 4 different environments (lens, air, glass, physiological solution), thus inducing optical distortions and measurement discrepancies. Moreover, due to the large strains observed locally (see next section), keeping the initial image at zero strain as the reference image for correlation computation did not ensure a systematic DIC algorithm convergence. The alternative was to update the reference image at each time step. Therefore, measurement errors were cumulated over the whole experiment, i.e., over the 25 images shot during the tensile test.

To estimate the overall strain discrepancies, an AF sample was used in testing conditions inside the thermo-regulated bath. The principle was to create rigid body motion by imposing a controlled displacement of the sample and compare it to the displacement measured at each point by the DIC procedure. Error amplitudes obtained in both planes and both directions are given in Tab. 1.

Using a similar procedure, strain measurement errors due to out-of-plane displacements were evaluated. In this case, the displacement imposed was per- 
Table 1: Estimation of strain errors resulting from optical distortions.

\begin{tabular}{|l|l|l|l|l|}
\hline & $E_{r r}^{\text {lamella }}$ & $E_{\theta \theta}^{\text {lamella }}$ & $E_{\theta \theta}^{\text {fibre }}$ & $E_{z z}^{\text {fibre }}$ \\
\hline Strain error amplitude [\%] & {$[-0.37,0.65]$} & {$[-0.82,0.58]$} & {$[-0.22,0.29]$} & {$[-0.44,0.75]$} \\
\hline
\end{tabular}

pendicular to the field of view in order to quantify the errors resulting from transverse strains that move the sample surface closer to or further from camera lens. The maximum transverse shrinkage and swelling measured on AF samples was used to impose the corresponding out-of-plane displacement. Over the whole set of tests, the maximal error was estimated at less than $0.09 \%$.

The various optical errors generate measurement uncertainties well described by a randomly distributed noise. Therefore, a filtering process was applied to displacement fields. It was based on the Savitsky-Golay algorithm using a quadratic function and a $5 \times 5$ points centred window. The final number of measurement points was $303 \pm 49$ in the fibre plane and $309 \pm 61$ in the lamella plane.

\section{Experimental results}

This work focuses on transverse mechanical behaviour in both directions, radial $\mathbf{e}_{r}$ and vertical $\mathbf{e}_{z}$. From the $19 \mathrm{AF}$ samples under investigation, complete strain fields are presented in 1 case and evolutions of average values are given for 4 cases that cover the whole range of observed behaviours. The fibre plane of fibres is given by circumferential and vertical directions, $\left(\mathbf{e}_{\theta}, \mathbf{e}_{z}\right)$, while the lamella plane of refers to circumferential and radial directions, $\left(\mathbf{e}_{\theta}, \mathbf{e}_{r}\right)$. 
3.1 Local strain fields

Using the image analysis procedure detailed in the previous section, an example of the strain fields identified in both planes at the end of tensile testing is given in Figs. 3 and 4. Strain fields computed from DIC measurements are superimposed over sample images. The white grid gives an indication of the overall deformation.

Note that circumferential strain fields (Fig. 3) are significantly heterogeneous and do not show any clear tendency. Local strain values range from $0 \%$ to $20 \%$ even though the macroscopic imposed strain is $10 \%$. This means that the average value is consistent while strong localization effects take place. Similar conclusions could be drawn for the whole set of experimental tests with local circumferential strain increasing to $30 \%$ in some cases.

For the same experimental test presented above, Fig. 4 shows transverse behaviours in both planes, i.e., vertical strain, $E_{z z}$, in the fibre plane and radial strain, $E_{r r}$, in the lamella plane. In the fibre plane, the strain field shows heterogeneous shrinkage similar in pattern and amplitude to that observed in the circumferential direction (Fig. 3a). These localization effects are thought to be associated with the complex, anisotropic micro-structure of AF tissue. The description of such effects is not addressed here since our objective is to analyse macroscopic homogenized behaviour.

Unlike previous strain fields, transverse behaviour in the lamella plane reveals some macroscopic features that are not associated with the microstructure. The strain field given in Fig. 4b clearly reveals a vertical strip with 


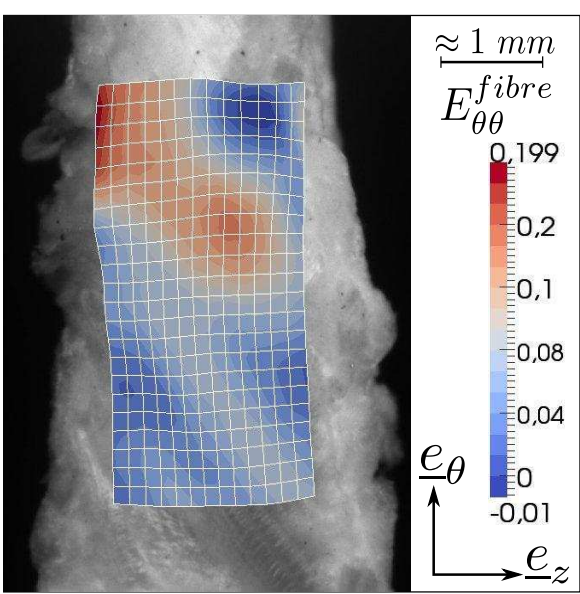

(a)

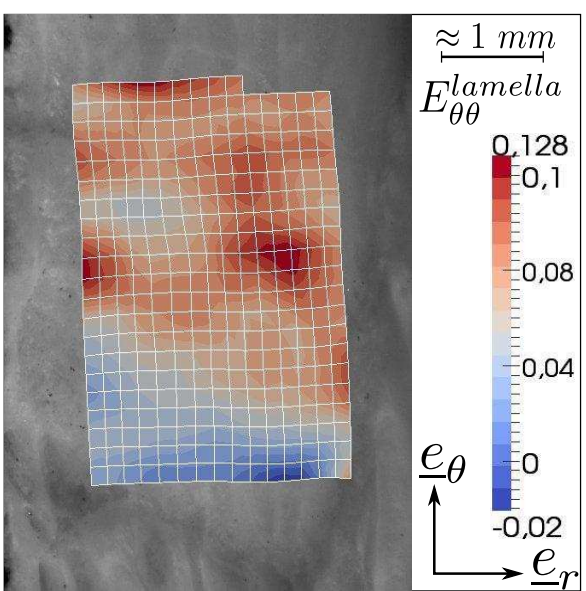

(b)

Fig. 3: Circumferential strain fields in the fibre plane and the lamella plane for a macroscopic circumferential strain imposed at $10 \%$

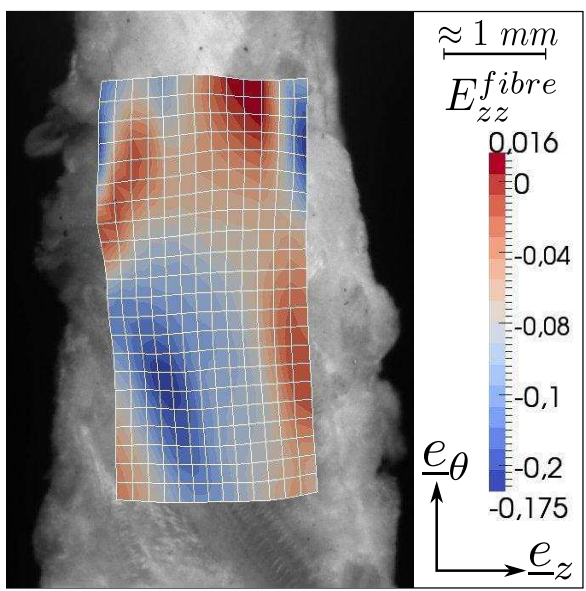

(a)

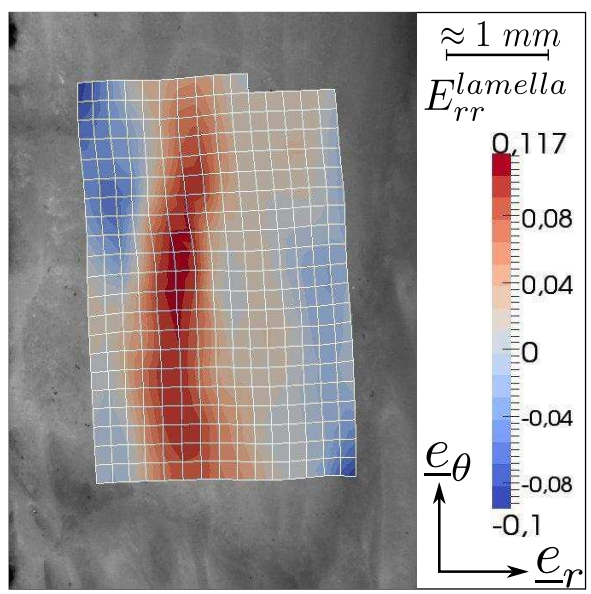

(b)

Fig. 4: Vertical and radial strain fields for a macroscopic circumferential strain imposed at $10 \%$ 
marked swelling in the central part. This swelling decreases monotonously to a slight shrinkage on both sides. This strain field is roughly symmetric with respect to the sample longitudinal axis. Strain values up to $42 \%$ can be observed locally.

Of the 19 samples under investigation, 12 presented such behaviour. The average value may have differed significantly from one test to another, but the overall shape of radial strain fields showed similar features, i.e., central part with a larger swelling or lower shrinkage and symmetric decreasing strain values towards each side of the sample. This issue will be discussed and interpreted in the last section.

\subsection{Evolution of average strain}

However, the large amount of data provided by DIC analysis can also be a source of difficulties. The strong heterogeneities observed in strain fields highlight localization effects linked to the complex micro-structure of AF tissue. Since our objective concerned macroscopic homogenized behaviour, average strain values were more suitable. Knowing that the measurement windows covers about $60 \%$ of the complete sample area, effective macroscopic strains could be slightly different from the average values calculated here. This could lead to some discrepancies in assessing macroscopic strain values.

The evolution of transverse strains, $E_{z z}$ in the fibre plane and $E_{r r}$ in the lamella plane, is given as function of the circumferential imposed strain $E_{\theta \theta}^{i m p}$, in Fig. 5. The 4 cases presented here were chosen to give an overview of the 
variety of behaviours that can be experimentally observed. The extreme cases represented in Figs. 5a and 5d give both limits for all experiments, while Figs. $5 \mathrm{~b}$ and $5 \mathrm{c}$ show intermediate cases. Indeed, the 4 cases presented show specific features that are observed in the whole set of tests.

Even if the meaning of Poisson's ratio is questionable when dealing with non-linear material, an apparent value can be defined as:

$$
\nu_{\theta z}=-\frac{E_{z z}}{E_{\theta \theta}} \quad \nu_{\theta r}=-\frac{E_{r r}}{E_{\theta \theta}}
$$

In case A (Fig. 5a), transverse shrinkage is observed in both planes. At the start of testing, both strain evolutions coincide. The slope at the origin of both curves is about $-0.5\left(\nu_{\theta z} \approx \nu_{\theta r} \approx 0.5\right)$ corresponding to the behaviour of an isotropic homogeneous incompressible material. This is thought to refer to the response of the background matrix when the fibres have not yet stretched and do not participate in the mechanical behaviour. Gradually, as the fibres tighten and play a predominant role in macroscopic mechanical behaviour, the curve associated with vertical strain $E_{z z}$ bends towards greater values while the radial strain $E_{r r}$ tends to a constant value around $2 \%$.

In case B (Fig. 5b), the bending of both curves is more pronounced and occurs early. It leads to a positive slope in the evolution of radial strain $E_{r r}$ at the end of tensile test. Indeed, a reversal from shrinkage to swelling is observed in the lamella plane. This reversal is far more apparent in case $\mathrm{C}$ (Fig. 5c) where the final radial strain is positive. Finally, in case D (Fig. 5d), the swelling previously observed in the lamella plane occurs from the start of the experiment reaching a positive strain value of $2.5 \%$ at the end. 


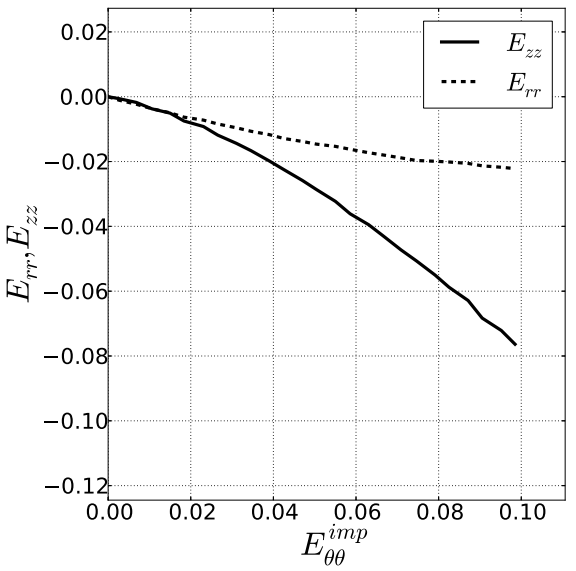

(a) case $\mathrm{A}$

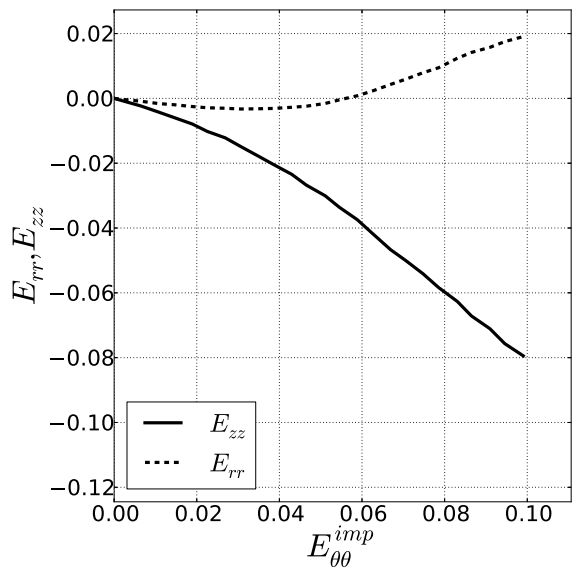

(c) case $\mathrm{C}$

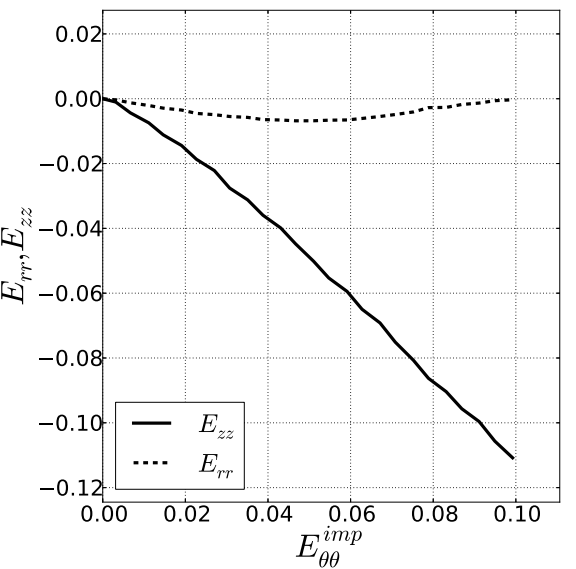

(b) case B

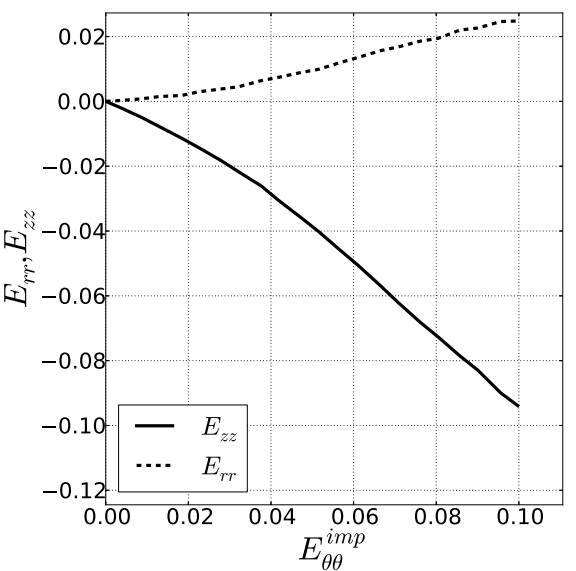

(d) case D

Fig. 5: Vertical and radial transverse strains as functions of the circumferential strain measured in 4 cases

These 4 cases highlight the fact that transverse behaviour in the fibre plane was quite similar for all 19 samples. The maximum average strain measured might have differed slightly from one sample to another, but the global evolu- 
tion was weakly non-linear and exhibited strong transverse shrinkage. Indeed, apparent Poisson's ratio was around $1\left(0.8 \leq \nu_{\theta z} \leq 1.1\right)$ which is outside the classical range valid for homogeneous isotropic material. Conversely, a wide range of transverse behaviour could be obtained in the lamella plane showing strong non-linear evolutions. In particular, the reversal from shrinkage to swelling was unusual. A key feature is that this reversal usually came with a bending of the vertical strain curve towards greater shrinkage.

\subsection{Relaxation tests}

Results from relaxation tests are synthesized in Figs 6 and 7. The first part $(t<100 s)$ corresponds to the tensile test already presented above while the second part $(100 s<t<1000 s)$ represents the relaxation phase per se. The time to equilibrium is fairly similar in each case while equilibrium is reached earlier in stress curves than in strain curves. It is noteworthy that, whatever the radial strain value obtained at the end of tensile testing (swelling or shrinkage), the relaxation phase is systematically associated with a decrease in radial strain.

Since vertical strain $\left(E_{z z}\right.$ in Fig 6$)$ remains nearly constant during relaxation phase, the decrease in radial strain leads to a global shrinkage. In the framework of poro-mechanics, this shrinkage necessarily results from a water loss and its kinetics is governed by tissue permeability. It means that an overpressure diffuses from sample to surrounding bath. The background matrix is well represented by isotropic poro-elastic behaviour. Thus, the local displace- 


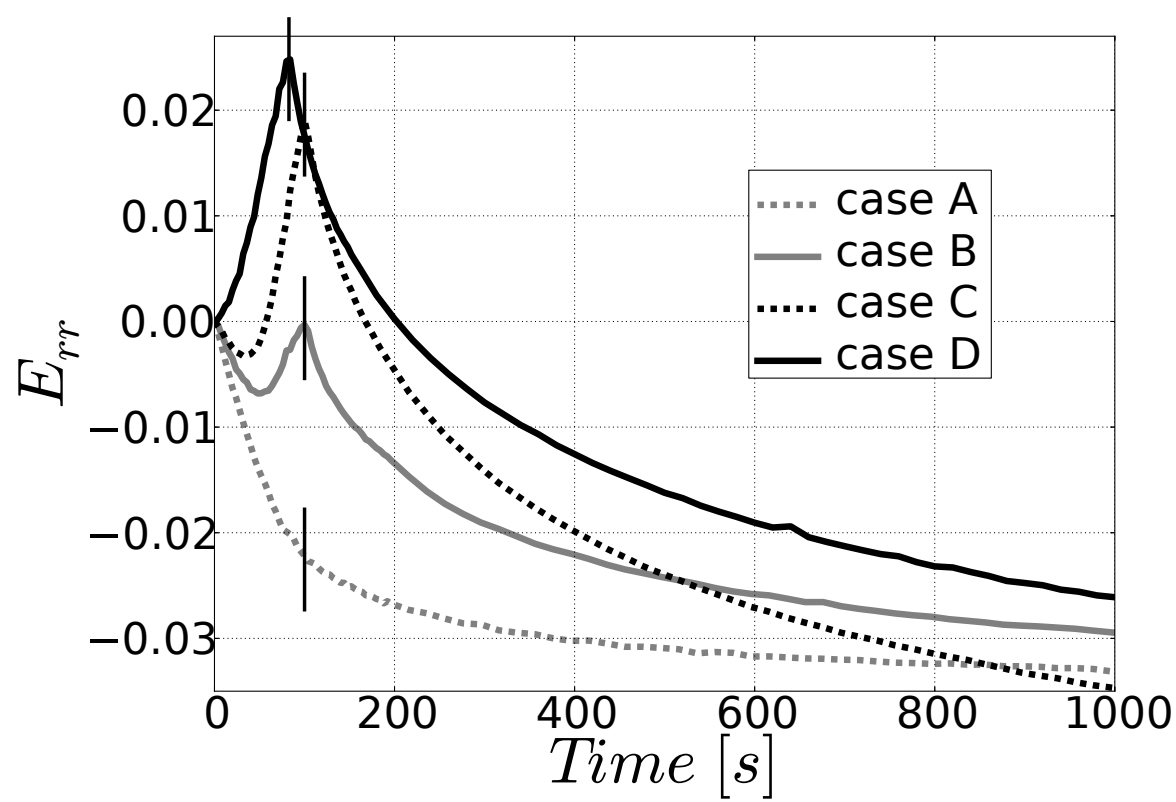

(a)

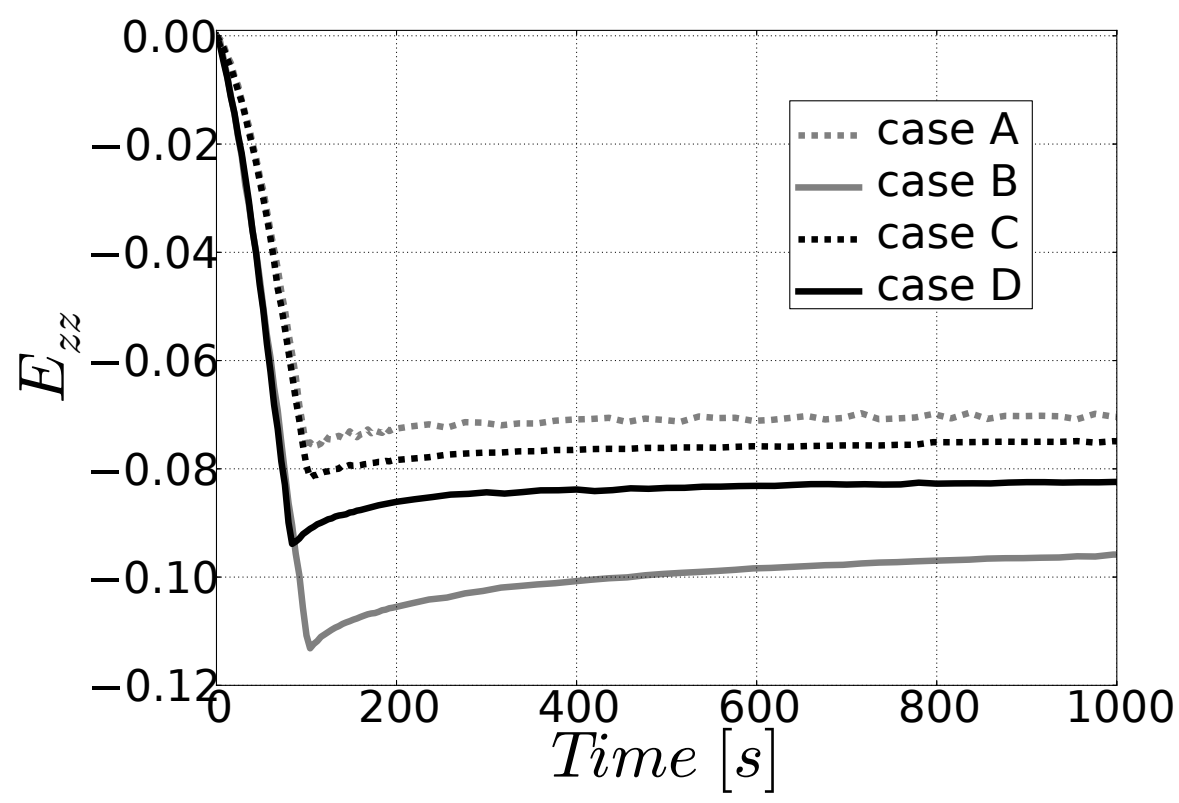

(b)

Fig. 6: a) Radial and b) vertical transverse strains versus time during tensile and relaxation tests 


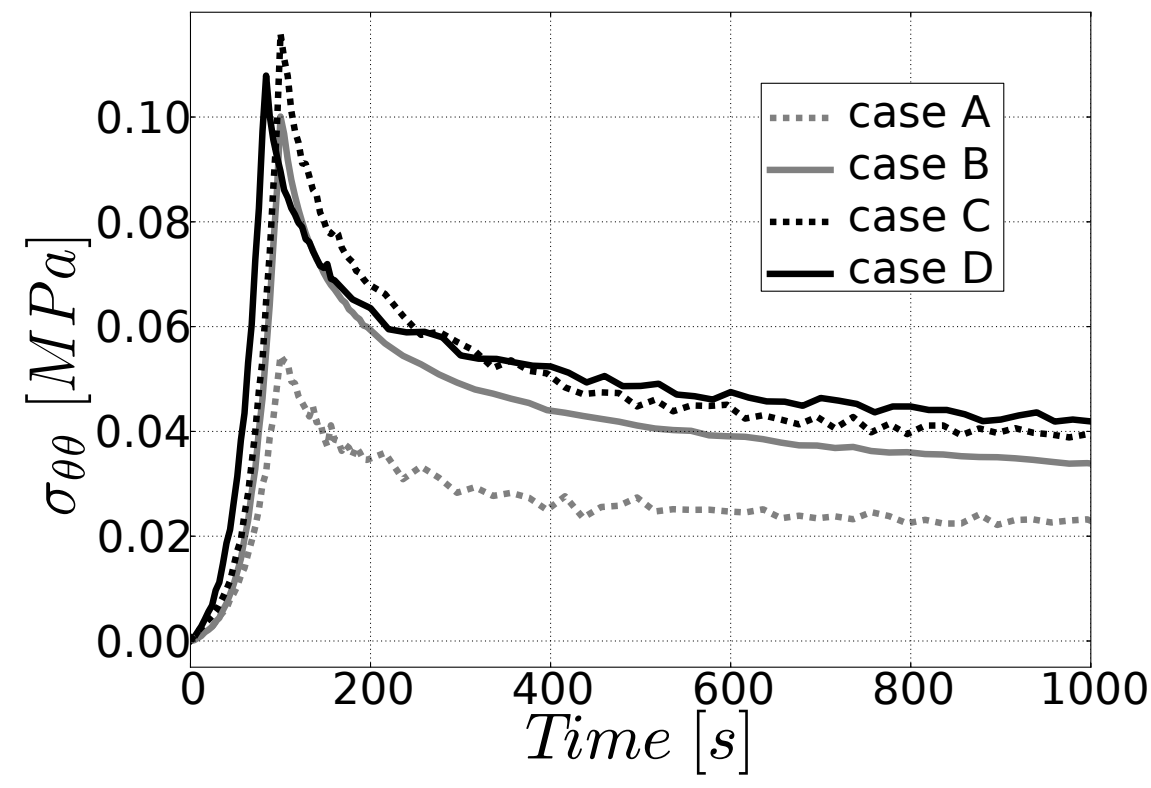

Fig. 7: Circumferential stress versus time during tensile and relaxation tests

ment vector $\mathbf{u}$ is described by the following equation [Coussy 2004]:

$$
\frac{\partial \mathbf{u}}{\partial t}+E K \Delta \mathbf{u}=0
$$

where $E$ is the Young modulus of the background matrix and $K$ its hydraulic permeability.

Neglecting the influence of glued ends on transverse liquid flows, the problem reduces to a bi-dimensional plane strain case. Initial condition is given by an homogeneous over-pressure and boundary conditions consist in free stress and pressure on each side while the vertical strain $E_{z z}$ is imposed to remain constant. Analytical solution of the evolution of radial strain $E_{r r}$ is given by [Carslaw and Jaeger 1959]:

$$
E_{r r}=E_{r r}^{\infty}+\left(E_{r r}^{0}-E_{r r}^{\infty}\right) \times \sum_{i=0}^{\infty} \frac{2}{\left(i+\frac{1}{2}\right)^{2} \pi^{2}} \exp \left[-\frac{1}{\left(i+\frac{1}{2}\right)^{2} \pi^{2}} \frac{t}{\tau}\right]
$$


where $E_{r r}^{0}$ and $E_{r r}^{\infty}$ are the radial strain at beginning and end of relaxation test. The characteristic time $\tau$ is defined by:

$$
\tau=\frac{1}{4 E K\left(\frac{1}{L_{r r}^{2}}+\frac{1}{L_{z z}^{2}}\right)}
$$

where $L_{r r}$ and $L_{z z}$ are the dimensions of the sample cross-section. The interest of such approach is to identify the characteristic time of relaxation which does not depend on initial conditions.

From the literature [Best et al 1994,Drost et al 1995, Iatridis et al 1998], the compressive modulus of the background matrix was evaluated to $E=$ $0.53 \mathrm{MPa}$. Using an inverse procedure based on Levenberg-Marquadt algorithm, the hydraulic permeability $K$ is determined from Eq. 4. Comparison between experimental and numerical relaxation tests are given in Fig. 8. Averaged over the 19 samples, the hydraulic permeability determined from this approach is $K=3.29 \times 10^{-15} \pm 1.66 \times 10^{-15} \mathrm{~m}^{4} \cdot \mathrm{N}^{-1} \cdot \mathrm{s}^{-1}$.

\section{Discussion}

Uniaxial tensile testing is a classical experimental technique to characterize the mechanical behaviour of biological tissue. When dealing with complex anisotropic material such as biological tissue, the stress/strain curve does not bring out sufficient data to identify material properties. Results can be enhanced by analysing transverse behaviour. With the potential offered by image analysis method, many difficulties can be overcome, especially those associated with short sample size and immersion in a physiological solution. Indeed, Dig- 


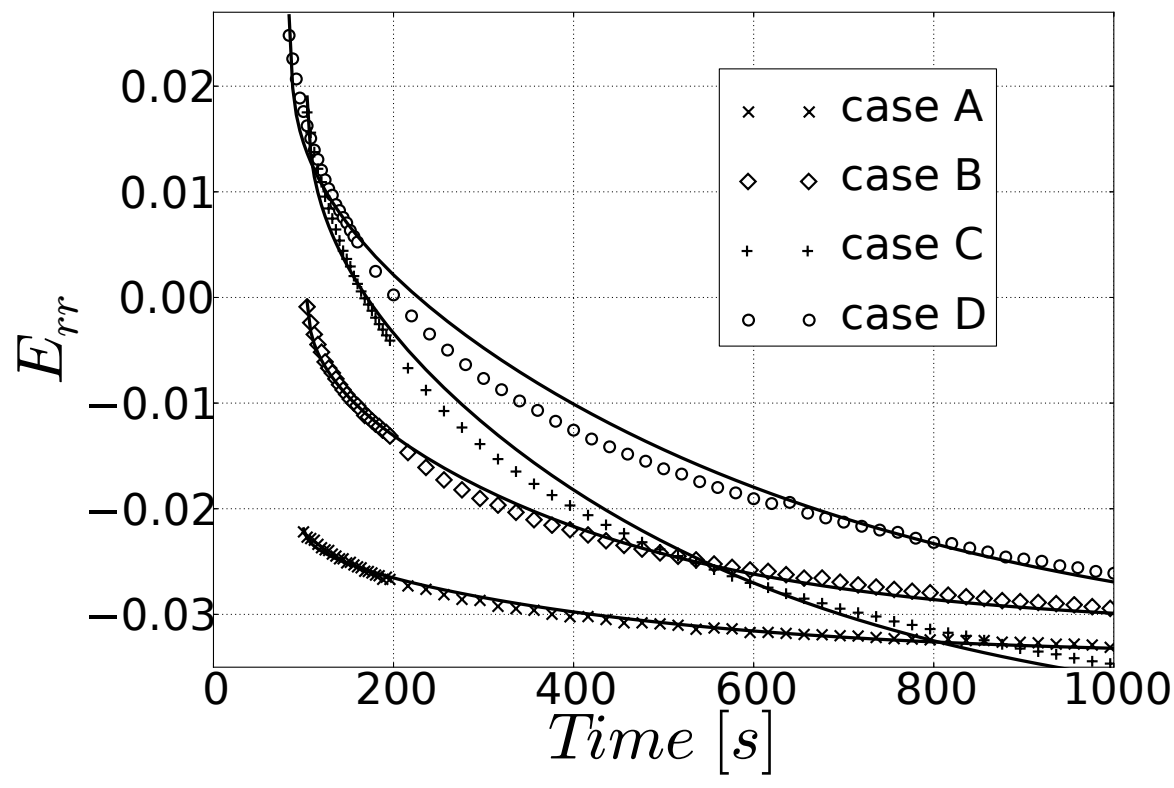

Fig. 8: Comparison between experimental and numerical radial strain relaxation versus time

ital Image Correlation techniques are capable of extracting a large amount of data (about 300 measurement points simultaneously in both planes) and providing bi-dimensional strain fields. The large number of measurement points makes it possible to focus on the central area of samples and avoid the side effects associated with irregular geometrical boundaries. Despite the weakly contrasted aspect of the natural AF pattern, the correlation algorithms converge efficiently. Taking optical distortions into account, strain absolute errors were estimated to be lower than $0.8 \%$ locally. When compared to the $10 \%$ circumferential strain imposed and to the $[-22 \%, 42 \%]$ strain range measured, DIC discrepancies cannot significantly influence the results nor hide partial in- 
formation. It therefore provides an efficient, accurate, non-invasive, low-priced and easy to implement alternative to standard measurement methods.

The millimetric scale of strain fields (measurement points distributed over a $0.18 \mathrm{~mm}$ grid) leads to heterogeneous displacement fields. Localization effects occur with a local maximum up to $30 \%$ while the global strain imposed is $10 \%$. The radial strain field (Fig. 4b) clearly gives prominence to a central part with a larger swelling than on both sides, i.e., strain distribution is symmetric with respect to the circumferential axis. This particular feature was observed in 12 of the 19 samples, specifically with samples showing behaviours between cases $\mathrm{B}$ and D (Figs $5 \mathrm{~b}$ and $5 \mathrm{~d}$ ). These 12 cases are characterized by the fact that the derivative $\frac{\partial E_{r r}}{\partial E_{\theta \theta}}$ is positive at the end of test representing a macroscopic tendency to swell (Fig. 5). In our view, this swelling behaviour cannot be attributed to any heterogeneities in tissue properties. For instance, the variation of fibre angle from external to internal $\mathrm{AF}$ is monotonous and cannot lead to the strain fields presented here.

This particular behaviour directly results from the porous nature of such biological tissue and its anisotropic stiffness. The collagen micro-structure provides high rigidities in the plane of fibres in both directions $\mathbf{e}_{\theta}$ and $\mathbf{e}_{z}$ while the background matrix leads to a lower rigidity in the radial direction [Elliott and Setton 2001,Holzapfel et al 2005]. The strong transverse shrinkage systematically observed in the fibre plane generates liquid over-pressure inside the samples. This over-pressure preferentially discharges in the radial direction where rigidity is lower, making the sample swell in the lamella plane. 
Moreover, this liquid pressure also induces outward liquid flows directed towards the sample free boundaries so that the pressure gradient must be symmetric and perpendicular to the sample sides. This leads to greater liquid pressure in the central part of samples which agrees with the distributions of radial strain experimentally observed (Fig. 4b).

At the beginning of tensile tests (Fig. 5a), isotropic homogeneous behaviour was generally recovered, similar to the response of a quasi-incompressible material. In the framework of saturated porous media, this corresponds to an undrained test where the characteristic time of pressure diffusion is much longer than the test duration [Coussy 2004]. Then, as the fibres uncrimp and tighten, transverse behaviours differentiate from each other. In the fibre plane, strain non-linearities were low and were roughly similar over the whole set of experiments. Indeed, transverse behaviour is mainly governed by the reorientation of fibres along the loading direction [Ambard and Cherblanc 2009]. However, in the lamella plane, non-linearities were much more pronounced where a reversal from shrinkage to swelling was observed in over half of the tests. The point of reversal corresponds to the apparition of the central strip observed in the radial strain field (Fig. 4b). This defines a transition zone from isotropic behaviour mainly governed by the background matrix to anisotropic behaviour controlled by the fibre network.

Poro-mechanical coupling effects were also underlined by relaxations tests (Fig. 7). Whatever the radial strain value at the end of tensile testing, radial strain decreased during relaxation phase. This observation agrees with 
the assertion that the liquid over-pressure diffuses towards the surrounding bath. Indeed, the greater the radial swelling the larger the strain relaxation. Thus, characteristic time of relaxation is governed by flow characteristic and this feature was used to estimate the tissue hydraulic permeability. The value obtained $K=3.29 \times 10^{-15} \mathrm{~m}^{4} \cdot \mathrm{N}^{-1} \cdot \mathrm{s}^{-1}$ is in agreement with previous results [Gu et al 1999, Yao et al 2002]. While being perfectible, this method is easy to implement. Further development will require a numerical model to account for the anisotropic micro-structure of AF tissue.

It must be recalled that these poro-mechanical couplings are transitory processes. The strain rate of $10^{-3} \mathrm{~s}^{-1}$ has a strong influence on experimental results. For instance in the literature, experimental tests carried out with AF tissue have been performed at strain rates of $10^{-4} s^{-1}$ [Acaroglu et al 1995, Bruehlmann et al 2004, Guerin and Elliott 2006, O'Connell et al 2012, Skaggs et al 1994, Wagner and Lotz 2004], $10^{-3} s^{-1}$ [Han et al 2012], $2.5 \times 10^{-3} s^{-1}$ [Smith et al 2008], $3 \times 10^{-3} s^{-1}$ [Tower et al 2002], $5 \times 10^{-3} s^{-1}$ [Fujita et al 1997], $25 \times 10^{-3} s^{-1}$ [Iatridis et al 2005]. In slower experimental tests $\left(\dot{E}_{\theta \theta}=10^{-4} s^{-1}\right)$, the loading phase runs over $1000 \mathrm{~s}$. While the circumferential stress seems to reach equilibrium before $t=1000 \mathrm{~s}$ (Fig. 7), this is not the case for radial strain (Fig. 6a). Moreover, the characteristic time of relaxation depends on the square of the cross-section dimensions (Eq. 4). This means that with larger samples, as is sometimes seen in the literature, the time required for equilibrium can be much greater. Therefore, a fair estimation of Poisson's ratios should rely 
on a careful analysis of transverse strains to distinguish between apparent and intrinsic values.

When dealing with non-linear material like AF tissue, mechanical characterization tests are very sensitive to initial conditions. For instance, the exponential strain potential usually developed to describe mechanical behaviour [Wagner and Lotz 2004, Bass et al 2004, Peng et al 2006, Guerin and Elliott 2007, O'Connell et al 2012] gives a prevailing role to the initial reference state. Any slight uncertainty concerning this initial state will have a significant impact on the material properties identified with experimental results [Sanchez et al 2012]. In this work, meticulous attention was paid to maintaining the initial conditions and imposing the same $0.05 N$ preload. Nevertheless, variability in sample dimensions $\left(L_{z z} \times L_{r r} \times L_{\theta \theta}=3.78 \pm 0.95 \times 3.67 \pm 0.67 \times 9.77 \pm 1.44 \mathrm{~mm}^{3}\right)$ led to non negligible differences regarding initial pre-stress. Bearing this in mind, the various curves presented can be assimilated to unique behaviour with different initial conditions. Indeed, case A (Fig. 5a) shows the response of an AF sample to weak pre-stress while case D (Fig. 5d) is associated to a stronger one. The two other cases B and C (Figs. 5b and 5c) are intermediate situations. This means that the uncertainties associated with initial conditions can be greater than those resulting from intrinsic inter-human variability [Tower et al 2002]. The average transverse strain in the lamella plane could be a fair indication of internal stress and should help to refine the definition of the initial state by proposing more discriminating criteria. Typically, 
the point where a reversal from shrinkage to swelling is observed might be used as the initial state to start a classical tensile test.

Using transverse strain fields besides the usual stress/strain curve should lead to enhancing the identification procedures based on numerical modelling. In particular, it brings out the transient behaviour associated with the porous nature. The work in progress concerns the development of an inverse procedure to identify both the mechanical and hydraulic properties of AF tissue using a single uniaxial tensile test.

\section{Conflict of interest statement}

There is no conflict of interest associated with the research carried out in the present paper.

\section{References}

[Acaroglu et al 1995] Acaroglu ER, Iatridis JC, Setton LA, Foster RJ, Mow VC, Weidenbaum M (1995) Degeneration and Aging Affect the Tensile Behavior of Human Lumbar Anulus Fibrosus. Spine 20(24):2690-2701

[Ambard and Cherblanc 2009] Ambard D, Cherblanc F (2009) Mechanical behavior of annulus fibrosus: a microstructural model of fibers reorientation. Ann Biomed Eng $37(11): 2256-2265$

[Argoubi and Shirazi-Adl 1996] Argoubi M, Shirazi-Adl A (1996) Poroelastic creep response analysis of a lumbar motion segment in compression. Journal of Biomechanics 29(10):1331 - 1339

[Baldit et al 2012] Baldit A, Ambard D, Cherblanc F, Royer P (2012) Chemo-hydromechanical coupling in annulus fibrosus tissue. Journal of Biomechanics 45, Supple- 
ment 1(0):S155, proceedings of ESB2012 - 18th Congress of the European Society of

Biomechanics

[Bass et al 2004] Bass EC, Ashford FA, Segal MR, Lotz JC (2004) Biaxial testing of human annulus fibrosus and its implications for a constitutive formulation. Ann Biomed Eng $32(9): 1231-1242$

[Best et al 1994] Best BA, Guilak F, Setton LA, Zhu W, Saed-Nejad F, Ratcliffe A, Weidenbaum M, Mow VC (1994) Compressive mechanical properties of the human anulus fibrosus and their relationship to biochemical composition. Spine (Phila Pa 1976) $19(2): 212-221$

[Bruehlmann et al 2004] Bruehlmann SB, Hulme PA, Duncan NA (2004) In situ intercellular mechanics of the bovine outer annulus fibrosus subjected to biaxial strains. J Biomech 37(2):223-231

[Carslaw and Jaeger 1959] Carslaw HS, Jaeger JC (1959) Conduction of Heat in Solids. Oxford Press

[Costi et al 2007] Costi JJ, Stokes IA, Gardner-Morse M, Laible JP, Scoffone HM, Iatridis JC (2007) Direct measurement of intervertebral disc maximum shear strain in six degrees of freedom: motions that place disc tissue at risk of injury. J Biomech 40(11):2457-2466

[Coussy 2004] Coussy O (2004) Poromechanics. John Wiley \& Sons

[Drost et al 1995] Drost MR, Willems P, Snijders H, Huyghe JM, Janssen JD, Huson A (1995) Confined compression of canine annulus fibrosus under chemical and mechanical loading. Journal of Biomechanical Engineering 117:390-396

[Elliott and Setton 2001] Elliott D, Setton L (2001) Anisotropic and inhomogeneous tensile behavior of the human anulus fibrosus: experimental measurement and material model predictions. Journal of Biomechanical Engineering 123:256-63

[Ferguson et al 2004] Ferguson SJ, Ito K, Nolte LP (2004) Fluid flow and convective transport of solutes within the intervertebral disc. J Biomech 37(2):213-221

[Fujita et al 1997] Fujita Y, Duncan NA, Lotz JC (1997) Radial tensile properties of the lumbar annulus fibrosus are site and degeneration dependent. Journal of Orthopaedic Research 15:814-819 
[Fujita et al 2000] Fujita Y, Wagner D, Biviji A, Duncan N, Lotz J (2000) Anisotropic shear behavior of the annulus fibrosus: effect of harvest site and tissue prestrain. Medical Engineering \&amp; Physics 22(5):349 - 357

[Grunhagen et al 2006] Grunhagen T, Wilde G, Soukane DM, Shirazi-Adl SA, Urban JP (2006) Nutrient supply and intervertebral disc metabolism. The Journal of Bone \& Joint Surgery 88(suppl 2):30-35

[Gu et al 1999] Gu WY, Mao XG, Foster RJ, Weidenbaum MD, Mow VC, Rawlins BA (1999) The Anisotropic Hydraulic Permeability of Human Lumbar Anulus Fibrosus. Influence of Age, Degeneration, Direction, and Water Content. Spine 24:2449-2455.

[Guerin and Elliott 2006] Guerin H, Elliott D (2006) Degeneration affects the fiber reorientation of human annulus fibrosus under tensile load. Journal of Biomechanics 39:14101418

[Guerin and Elliott 2007] Guerin H, Elliott D (2007) Quantifying the Contributions of Structure to Annulus Fibrosus Mechanical Function Using a Nonlinear, Anisotropic, Hyperelastic Model. Journal Orthopaedic Research 25:508-516

[Han et al 2012] Han W, Nerurkar N, Smith L, Jacobs N, Mauck R, Elliott D (2012) Multiscale Structural and Tensile Mechanical Response of Annulus Fibrosus to Osmotic loading. Annals of Biomedical Engineering 40:1610-1621

[Holzapfel et al 2005] Holzapfel GA, Schulze-Bauer CAJ, Feigl G, Regitnig P (2005) Single lamellar mechanics of the human lumbar anulus fibrosus. Biomechanics and Modeling in Mechanobiology 3:125-140

[Huang et al 2001] Huang CY, Mow V, Ateshian G (2001) The role of flow-independent viscoelasticity in the biphasic tensile and compressive responses of articular cartilage. Journal of Biomechanical Engineering 123:410-417

[Huyghe and Jongeneelen 2012] Huyghe J, Jongeneelen C (2012) 3d non-affine finite strains measured in isolated bovine annulus fibrosus tissue samples. Biomechanics and Modeling in Mechanobiology 11:161-170

[Iatridis et al 1997] Iatridis J, Setton L, Weidenbaum M, Mow V (1997) The viscoelastic behavior of the non-degenerate human lumbar nucleus pulposus in shear. Journal of 
Biomechanics 30:1005-1013

[Iatridis et al 1998] Iatridis J, Setton L, Foster R, Rawlins B, Weidenbaum M, Mow V (1998) Degeneration affects the anisotropic and nonlinear behaviors of human anulus fibrosus in compression. Journal of Biomechanics 31:535-544

[Iatridis et al 2005] Iatridis JC, MaClean JJ, Ryan DA (2005) Mechanical damage to the intervertebral disc annulus fibrosus subjected to tensile loading. J Biomech 38(3):557565

[Klisch and Lotz 1999] Klisch SM, Lotz JC (1999) Application of a fiber-reinforced continuum theory to multiple deformations of the annulus fibrosus. J Biomech 32(10):10271036

[Magnier et al 2009] Magnier C, Boiron O, Wendling-Mansuy S, Chabrand P, Deplano V (2009) Nutrient distribution and metabolism in the intervertebral disc in the unloaded state: a parametric study. J Biomech 42(2):100-108

[Malandrino et al 2011] Malandrino A, Noailly J, Lacroix D (2011) The effect of sustained compression on oxygen metabolic transport in the intervertebral disc decreases with degenerative changes. PLoS Comput Biol 7(8):e1002,112

[Michalek et al 2009] Michalek AJ, Buckley MR, Bonassar LJ, Cohen I, Iatridis JC (2009) Measurement of local strains in intervertebral disc anulus fibrosus tissue under dynamic shear: Contributions of matrix fiber orientation and elastin content. Journal of Biomechanics 42(14):2279-2285

[Michalek et al 2010] Michalek AJ, Buckley MR, Bonassar LJ, Cohen I, Iatridis JC (2010) The effects of needle puncture injury on microscale shear strain in the intervertebral disc annulus fibrosus. The Spine Journal 10(12):1098 - 1105

[O'Connell et al 2009] O'Connell GD, Guerin HL, Elliott DM (2009) Theoretical and uniaxial experimental evaluation of human annulus fibrosus degeneration. Journal of biomechanical engineering 131

[O'Connell et al 2012] O'Connell G, Sen S, Elliott D (2012) Human annulus fibrosus material properties from biaxial testing and constitutive modeling are altered with degeneration. Biomechanics and Modeling in Mechanobiology 11:493-503 
[Peng et al 2006] Peng X, Guo Z, Moran B (2006) An anisotropic hyperelastic constitutive model with fiber-matrix shear interaction for the human annulus fibrosus. Journal of Applied Mechanics 73:815-824

[Rannou et al 2000] Rannou F, Poiraudeau S, Corvol M, Revel M (2000) Biochimie et biologie du disque intervertbral. Rev Rhum [d Fr] 67suppl4:214-216

[Sanchez et al 2012] Sanchez M, Ambard D, Costalat V, Mendez S, Jourdan F, Nicoud F (2012) Biomechanical assessment of the individual risk of rupture of cerebral aneurysms: A proof of concept. Annals of Biomedical Engineering pp 1-13

[Shirazi-Adl 1989] Shirazi-Adl A (1989) Strain in fibers of a lumbar disc. analysis of the role of lifting in producing disc prolapse. Spine (Phila Pa 1976) 14(1):96-103

[Silva et al 2005] Silva P, Crozier S, Veidt M, Pearcy M (2005) An experimental and finite element poroelastic creep response analysis of an intervertebral hydrogel disc model in axial compression. Journal of Materials Science: Materials in Medicine 16:663-669

[Skaggs et al 1994] Skaggs D, Weidenbaum M, Iatridis J, Ratcliffe A, Mow V (1994) Regional variation in tensile properties and biochemical composition of the human lumbar anulus fibrosus. Spine 19(12):1310-1319

[Smith et al 2008] Smith L, Byers S, Costi J, Fazzalari N (2008) Elastic Fibers Enhance the Mechanical Integrity of the Human Lumbar Anulus Fibrosus in the Radial Direction. Annals of Biomedical Engineering 36:214-223

[Swider et al 2010] Swider P, Pédrono A, Ambard D, Accadbled F, de Gauzy JS (2010) Substructuring and poroelastic modelling of the intervertebral disc. Journal of Biomechanics 43:1287-1291

[Tower et al 2002] Tower TT, Neidert MR, Tranquillo RT (2002) Fiber alignment imaging during mechanical testing of soft tissues. Annals of Biomedical Engineering 30:12211233

[Wagner and Lotz 2004] Wagner DR, Lotz JC (2004) Theoretical model and experimental results for the nonlinear elastic behavior of human annulus fibrosus. Journal Orthopaedic Research 22:901-909 
[Wattrisse et al 2001] Wattrisse B, Chrysochoos A, Muracciole JM, Nmoz-Gaillard M (2001) Analysis of strain localization during tensile tests by digital image correlation. Experimental Mechanics 41(1):29-39

[Woods et al 2010] Woods B, Sowa G, Vo N, Kang J (2010) A change in strategy: the use of regenerative medicine and tissue engineering to augment the course of intervertebral disc degeneration. Operative Techniques in Orthopaedics 20(2):144-153

[Yao et al 2002] Yao H, Justiz M, Flagler D, Gu W (2007) Effects of Swelling Pressure and Hydraulic Permeability on Dynamic Compressive Behavior of Lumbar Annulus Fibrosus. Annals of Biomedical Engineering 30:1234-1241

[Yao and Gu 2007] Yao H, Gu W (2007) Three-dimensional inhomogeneous triphasic finiteelement analysis of physical signals and solute transport in human intervertebral disc under axial compression. Journal of Biomechanics 40:2071-2077 